\title{
Smooth Optimal Quantum Control for Robust Solid-State Spin Magnetometry
}

\author{
Tobias Nöbauer, ${ }^{1, *}$ Andreas Angerer, ${ }^{1}$ Björn Bartels, ${ }^{2}$ Michael Trupke, ${ }^{1}$ Stefan Rotter, ${ }^{3}$ \\ Jörg Schmiedmayer, ${ }^{1}$ Florian Mintert, ${ }^{2,4}$ and Johannes Majer ${ }^{1}$ \\ ${ }^{1}$ Vienna Center for Quantum Science and Technology and Atominstitut, Vienna University of Technology (TU Wien), \\ Stadionallee 2, 1020 Vienna, Austria \\ ${ }^{2}$ Freiburg Institute for Advanced Studies, Albert-Ludwigs-Universität Freiburg, Albertstraße 19, 79104 Freiburg, Germany \\ ${ }^{3}$ Institute for Theoretical Physics, Vienna University of Technology (TU Wien), Wiedner Hauptstraße 8-10/136, 1040 Vienna, Austria \\ ${ }^{4}$ QOLS, Blackett Laboratory, Imperial College, London SW7 2AZ, United Kingdom
}

(Received 9 June 2015; published 6 November 2015)

\begin{abstract}
We experimentally demonstrate a simple yet versatile optimal quantum control technique that achieves tailored robustness against qubit inhomogeneities and control errors while requiring minimal bandwidth. We apply the technique to nitrogen-vacancy (NV) centers in diamond and verify its performance using quantum process tomography. In a wide-field NV center magnetometry scenario, we achieve a homogeneous sensitivity across a 33\% drop in control amplitude, and we improve the sensitivity by up to 2 orders of magnitude for a normalized detuning as large as $40 \%$, achieving a value of $20 \mathrm{nT} \mathrm{Hz}^{-1 / 2} \mu \mathrm{m}^{3 / 2}$ in sensitivity times square root volume.
\end{abstract}

DOI: 10.1103/PhysRevLett.115.190801

PACS numbers: 07.55.Ge, 02.30.Yy, 61.72.jn, 81.05.ug

Introduction.-The practical operation of quantum devices - such as quantum-enabled sensors, quantum processors, and memories - requires advanced quantum optimal control techniques that are robust with respect to experimental and material imperfections, lack of knowledge about device characteristics, control errors, and noise. Often, qubits are placed into high- $Q$ cavities, e.g., in circuit QED-based memories [1,2] and photonic quantum networks [3], in order to facilitate coherent information transfer. Since high quality factors imply small bandwidth, control techniques with well-defined narrow bandwidth as well as low power requirements are desirable. In scaled-up solid-state quantum systems, parameters are often hard to control (e.g., when implanting ions into crystals), to characterize on a large scale, and to tune. General techniques for coping with these stringent requirements are (quantum) optimal control algorithms [4] such as Krotov [5], GRAPE [6] and CRAB [7] whose effectiveness for pulse shaping has been demonstrated in a number of fields, ranging from NMR [6] to ultracold atoms [8]. For defect center qubits, similar techniques have been shown to improve fidelities of strongly driven spin flips [9] and entangling gates in nitrogen-vacancy (NV)-nuclear spin quantum registers [10].

In this Letter we demonstrate a simple yet highly customizable quantum optimal control approach [11] capable of achieving extensive robustness and high fidelity even with low control bandwidth and power. To this end, we design and evaluate control pulses suitable for enhancing sensitivity

Published by the American Physical Society under the terms of the Creative Commons Attribution 3.0 License. Further distribution of this work must maintain attribution to the author(s) and the published article's title, journal citation, and DOI. and homogeneity in nitrogen-vacancy (NV) center-based sensing modalities [12-15]. We use pulse design in frequency space $[11,16-18]$ since it permits us to easily construct spectrally narrow pulses. We parametrize the control pulse in terms of a small set of time-periodic functions (typically, eight to ten), and we perform variational analysis in Floquet space to optimize these parameters to meet robustness and fidelity requirements. The fact that the resulting pulses do not contain frequencies higher than the first few overtones of the pulse duration eases their experimental implementation by minimizing ringing and reflection effects. The numerical optimization is computationally efficient and can be extended to designing multiqubit gates and optimizing for other objectives and constraints—such as pulse duration [11] — making it suitable for a wide range of applications in quantum control of ensemble-based or multiqubit quantum information processors.

Pulse design for NV center magnetometry. For sensing architectures based on $\mathrm{NV}$ ensembles, high defect densities increase the fluorescence yield but come at the expense of increased inhomogeneous broadening due to irradiation damage in the host crystal, as well as of a decreased optical read-out contrast [19-22]. In scenarios involving wide-field magnetic imaging using ensembles of NVs [23,24], additional complications arise: Inhomogeneities in the control field amplitude as well as detunings due to inherent or extrinsic inhomogeneous broadening (which can reach several megahertz in high-density samples) reduce the control fidelity. These complications become particularly severe for randomly oriented NV centers in nanodiamonds.

For magnetometry with high-density, inhomogeneously broadened NV ensembles, we optimize the control pulses for robustness with respect to control amplitude variations 
(a)

(b)
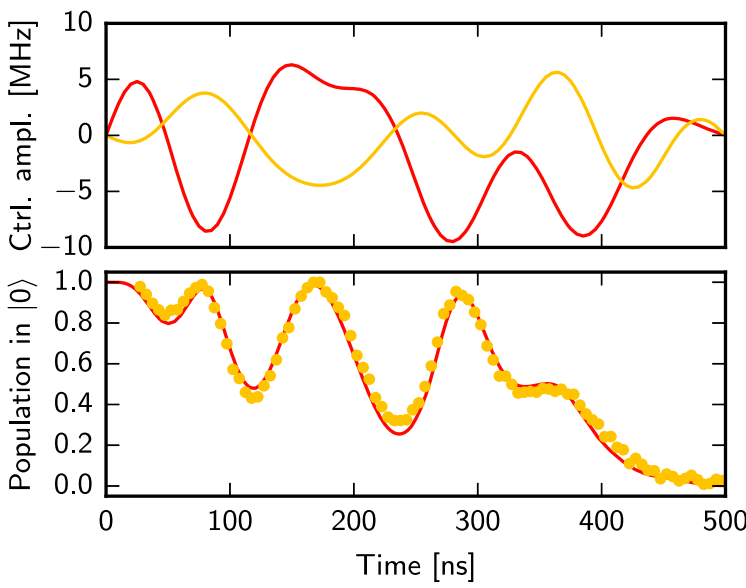

FIG. 1 (color online). Qubit evolution during a smooth optimal control pulse. (a) In-phase (red line) and quadrature (orange line) control amplitude components of a smooth control pulse implementing a robust state transfer from $|0\rangle$ to $|1\rangle$. The pulse consists of ten harmonics $(1,2, \ldots, 10 \mathrm{MHz})$ and uses a maximum control amplitude (Rabi frequency) of $9.5 \mathrm{MHz}$. It is optimized to be robust with respect to a $\pm 25 \%$ variation in control amplitude and a Gaussian distribution of detunings with $8 \mathrm{MHz}$ FWHM. (b) Simulated (solid line) and measured (dots) evolution of the qubit population in $|1\rangle$ during playback of the pulse shown in (a). The simulation shows coherent evolution in the rotating wave approximation.

of $\pm 25 \%$ and detunings (inhomogeneous linewidth) as high as $\pm 4 \mathrm{MHz}$. We constrain the control amplitudes occurring in the pulse to the maximum Rabi frequency achievable using our equipment, i.e., 10 to $20 \mathrm{MHz}$ (cf. the Supplemental Material [25]). A typical resulting control pulse is shown in Fig. 1(a) (the solid line): Consisting of only ten frequency components with constant in-phase and quadrature amplitude coefficients, this pulse is designed to implement a state transfer from state $|0\rangle$ to $|1\rangle$ with a theoretical infidelity (defined as one minus the population in $|1\rangle$ after the pulse) not larger than one percent within the robustness windows specified above. If experimental hardware permits one to realize shorter and stronger pulses, one benefits from the fact that the relevant figure of merit is the product of inhomogeneous broadening and pulse duration. Reducing the pulse duration thus renders the control problem less challenging and permits us to further reduce the frequencies in the control pulse or to include additional optimization goals, such as robustness with respect to pulse playback imperfections.

Experimental verification of design goals.-In order to verify its properties, we now apply the pulse shown in Fig. 1(a) to an effective qubit consisting of the $\left|m_{S}=0\right\rangle$ and $\left|m_{S}=-1\right\rangle$ states of a single NV center by modulating the two control amplitude components onto a resonant carrier microwave. Since the hyperfine structure caused by the ${ }^{14} \mathrm{~N}$ nuclear spin associated with the NV center would have complicated the dynamics, we polarize the nuclear spin by optical pumping in a static magnetic field of $\approx 0.50 \mathrm{mT}$ aligned with the $\mathrm{NV}$ center symmetry axis [31]. We interrupt the pulse at a series of time offsets and optically read-out the resulting spin projection of the NV center, resulting in the data shown in Fig. 1(b). We find very good agreement with the expected behavior as simulated using a simple two-level Hamiltonian (see the Supplemental Material [25]).

Using the same polarization and read-out scheme, we now proceed to experimentally verify that the pulse is indeed robust with respect to the detuning range (inhomogeneous broadening) and variations of the control amplitude that were required in the design procedure. To do so, we apply the full pulses and scan the carrier frequency over a range of $\pm 10 \mathrm{MHz}$ across resonance and vary the control amplitude by $\pm 50 \%$ with respect to the central value for which the pulse was designed. The result is shown in Fig. 2 in comparison to the theoretical fidelity landscape, which is in excellent agreement with the data, only limited by the photon shot noise inherent to the optical spin read-out. For comparison, we performed a simulation and experiment with a rectangular pulse of the same maximum control amplitude. It is apparent that the demanding robustness requirements relevant for NV-ensemble-based sensing are met and the results greatly increase fidelities over a wide range of detunings (as much as $\pm 80 \%$ of the resonant Rabi frequency) and control amplitudes (up to $\pm 40 \%$ of the correct value). Our pulses also outperform basic composite pulses [32] such as the $(\pi / 2)_{y}-\pi_{x}-(\pi / 2)_{y}$ and other variable rotation sequences (cf. the Supplemental Material [25]).

In our experiments discussed so far, we prepared the qubit in a well-defined initial state $\left(\left|m_{S}=0\right\rangle\right)$ before applying the control pulses; the pulses we examined merely transferred the system from one specific initial state to one final state. However, in interferometric sensing schemes (based on Ramsey or spin-echo sequences) as well as in general quantum information processing tasks, it is imperative that the pulses implement a specific time evolution, i.e., propagator, and not just a transfer from one specific state to another (which could be achieved using many different propagators). Experimentally, the full system evolution can be tracked using quantum process tomography [33]: Each member of a complete set of basis states is prepared, the operation under test is applied to each basis state, and the result is characterized by state tomography (see the Supplemental Material [25]). When performing this experiment with a $\pi_{x}$ pulse on resonance and with an ideal control amplitude, we obtain a process matrix that agrees with the ideal result at a fidelity of $99 \%$ (see Fig. 3). Remarkably, when repeating the tomography with the same pulse at a detuning of $8 \mathrm{MHz}$, the fidelity decreases only slightly, to a value of $93 \%$. When using the same pulse scaled to $87.5 \%$ of the ideal amplitude and at no detuning, we measure a fidelity of $92 \%$. (Theoretical values obtained from a two-level Hamiltonian simulation are $92 \%$ for both 
(a)

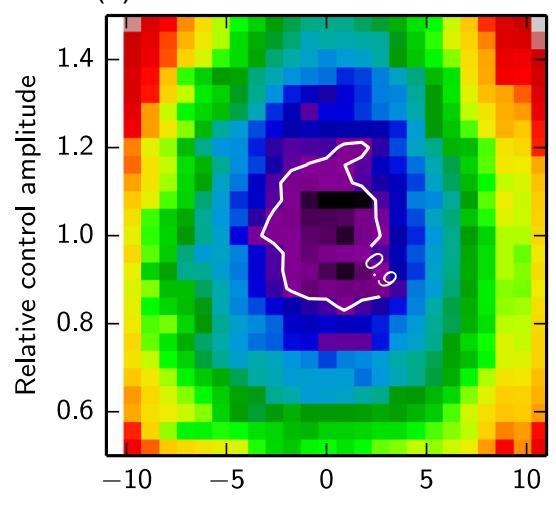

(b)

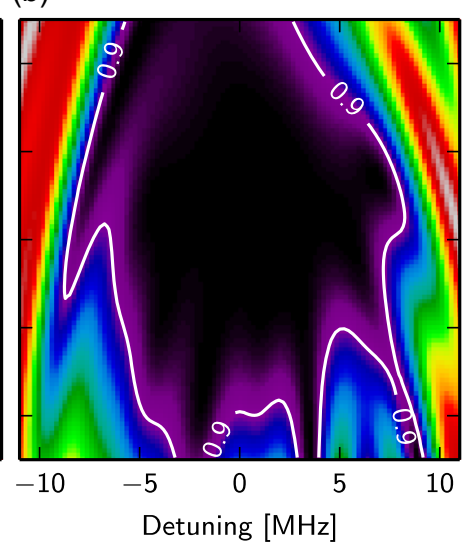

(c)

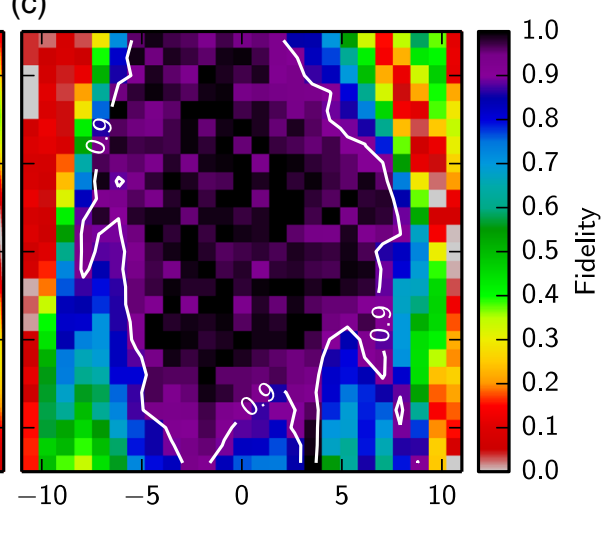

FIG. 2 (color online). State transfer fidelity of rectangular and optimal pulses compared. (a) Measured state transfer fidelity for a rectangular pulse using the same maximum control amplitude as the optimal pulse. (b) Simulated and (c) measured state transfer fidelity for a range of detunings and control amplitude scalings for the optimal pulse shown in Fig. 1(a). White lines are contour lines at fidelity 0.9. The range of detunings shown here corresponds to $\approx \pm 110 \%$ of the maximum resonant Rabi frequency used in the pulses $(9.5 \mathrm{MHz})$. Since optical spin read-out is limited by photon shot noise, exceedingly long integration times would be required to directly observe the very high theoretical fidelities in the experiment. The shape of the fidelity landscape, however, is reproduced clearly in the data.

the detuned and the amplitude-scaled optimal pulse. Simulated values for rectangular pulses of the same maximum Rabi frequency are $85 \%$ and $84 \%$, respectively).

Magnetometry using smooth optimal control.-Having shown the robustness and fidelity of our control pulses using a single NV center, we are now in a position to apply them to the system that they were designed for-an ensemble of inhomogeneously broadened NVs-and perform magnetic sensing. We use a layer of NV centers (thickness $8 \mathrm{~nm}$ ), which was formed $12 \mathrm{~nm}$ below the surface of a chemical vapor deposition diamond of high chemical purity by nitrogen ion implantation. The NV ensemble has a linewidth of $960 \mathrm{kHz}$ (FWHM). We perform ac magnetometry by repeatedly initializing the NV center spins in the $\left|m_{S}=0\right\rangle$ state by nonresonant optical pumping, driving a spin-echo sequence with fixed free precession time $\tau$ and reading out the spin projection via optically detected magnetic resonance, akin to the protocol used in earlier work [13]. To characterize our magnetometer, we deliberately apply an ac magnetic field of period $2 \tau$ in phase with the spin-echo sequence and scan its amplitude $B$. This causes a modulation of the spin-echo amplitude which serves as the magnetometric signal $S(B) \propto \cos g \mu_{B} B$, where $g \approx 2$ is the NV center electron $g$ factor, and $\mu_{B}$ is the Bohr magneton. From the slope $\delta S / \delta B$ of the signal and the noise level, we extract the sensitivity, which we plot in Fig. 4. For comparison, we performed the experiment with simple, rectangular control pulses, as well as with optimal pulses. In order to emulate conditions typical for wide-field sensing geometries, we scan the detuning of our ensemble as well as the control amplitude across a range of values.

While for the optimal pulses the sensitivity remains constant to within $30 \%$ across the recorded parameter range, for the rectangular pulses it drops off by 1 to 2 (a)

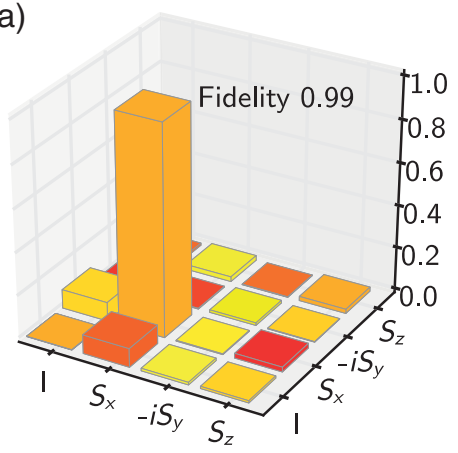

(b)

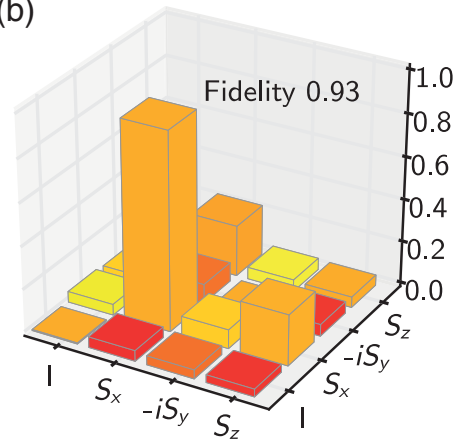

(c)

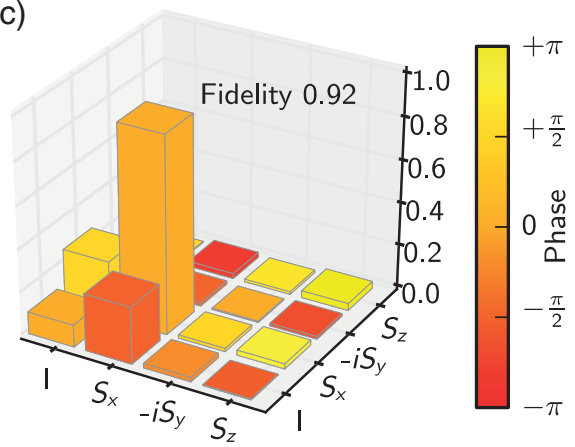

FIG. 3 (color online). Robustness of a single qubit gate implemented by a smooth optimal control pulse characterized by quantum process tomography. Process tomography of a $\pi_{x}$ smooth optimal control pulse for (a) correct (100\%) control amplitude and no detuning, (b) correct control amplitude and $8 \mathrm{MHz}$ detuning, and (c) $87.5 \%$ of the correct control amplitude and no detuning. Maximum resonant Rabi frequency used in the pulse: $20 \mathrm{MHz}$. See the Supplemental Material [25] for the measurement scheme and details of the analysis. 

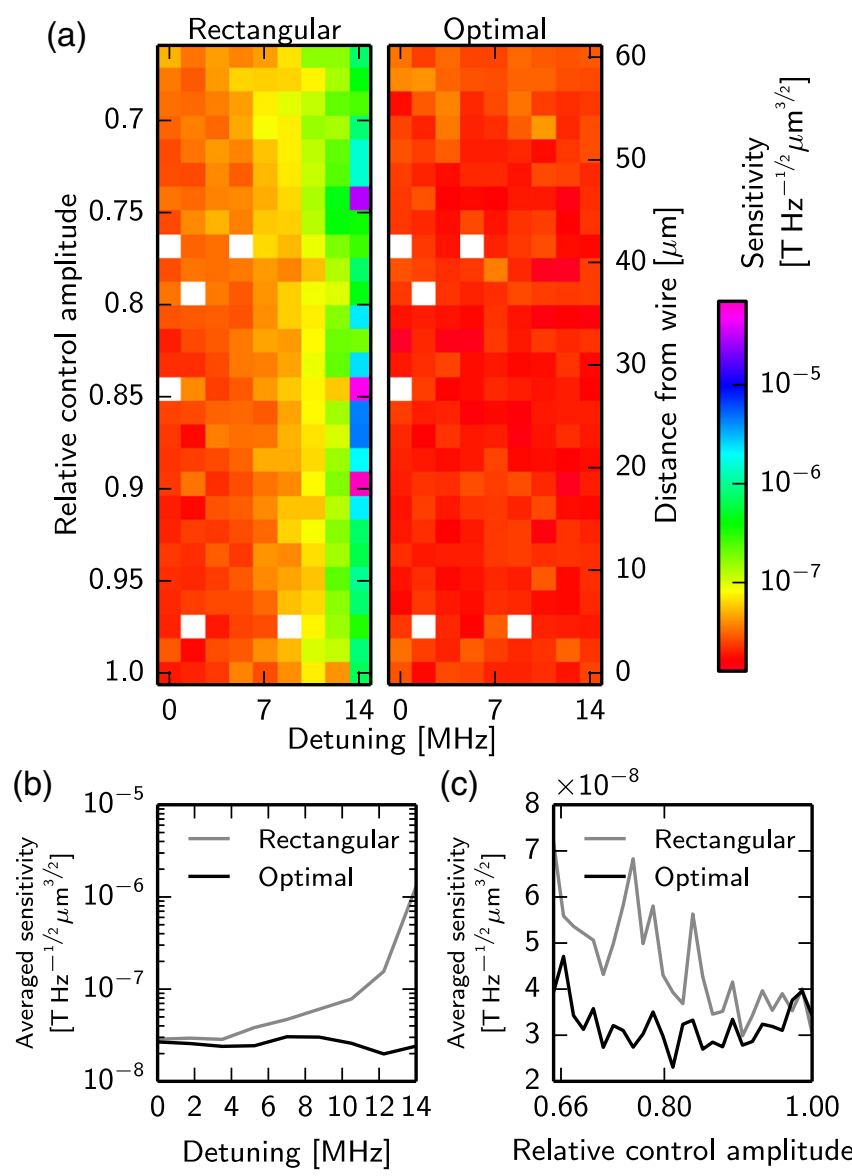

FIG. 4 (color online). Improved magnetometric sensitivity under conditions akin to wide-field sensing. (a) Measured ensemble-based sensitivity times root volume of a spin-echo ac magnetometry scheme using rectangular pulses (left panel) and smooth optimal pulses (right panel) for a range of detunings and control amplitude scalings. Note the logarithmic color scale. White pixels indicate data points that were discarded due to experimental instabilities. The smooth pulse sequence consisted of a $|0\rangle \rightarrow(|0\rangle+|1\rangle) / \sqrt{2}$ state transfer pulse, followed by a phase collection time $\tau=1.2 \mu \mathrm{s}$, a $\pi_{y}$ pulse, collection time $\tau$, and a final $(\pi / 2)_{y}$ pulse. The right vertical axis indicates approximate distances from the antenna wire $(100 \times 20 \mu \mathrm{m})$ generating the control fields (data obtained from a linear fit to measurements of relative Rabi frequency vs distance from wire). (b) Data from (a) integrated along the vertical axis and (c) horizontal axis. See the Supplemental Material [25] for pulse design parameters and measurement details.

orders of magnitude as the detuning becomes larger (i.e., for NV centers further away from a bias wire in wide-field magnetometry, or for broad ensembles). Similarly, but less pronounced, we observe a larger drop in sensitivity at large control amplitude mismatches for the rectangular pulses than for the optimal pulses. All sensitivity values are corrected for count rate limitations of our setup (see the Supplemental Material [25]). Using optimal control, we obtain a peak magnetometric sensitivity times root volume of $13 \mathrm{nT} \mathrm{Hz}^{-1 / 2} \mu \mathrm{m}^{3 / 2}$, which is comparable to reported values for room temperature ensemble-based sensing using off-resonant read-out $[23,34]$. We note that using optimal control not only allows for homogeneous sensitivity but also increases the peak sensitivity by $35 \%$ in our sample. The absolute magnetic sensitivity achievable with an NV ensemble is inversely proportional to the square root [35] of its dephasing time $T_{2}$ and depends on a number of material parameters [21]. In our experiments, we have $T_{2}=2.2 \mu \mathrm{s}$, which can still be greatly extended using advanced growth techniques [23]. Dynamical decoupling has been shown to increase single-NV center magnetic sensitivity [36] and may be combined with our technique.

To summarize, we have demonstrated how a high magnetic sensitivity in NV-ensemble magnetometry can be maintained over wide ranges of control parameters. For highly broadened or detuned ensembles, we improve per-volume sensitivity by up to 2 orders of magnitude. Our strategy builds on greatly reducing control errors due to inhomogeneous broadening and control amplitude variations using smooth, robust optimal control pulses. The absolute sensitivity achieved in our experiments is limited by the density of the NV centers, their dephasing time, and the read-out contrast, all of which can be further improved by optimizing implantation and material parameters. The demonstrated ability to compensate for residual system imperfections in a simple and robust manner, together with the anticipated improvement in system preparation, thus promises to provide the basis for applications satisfying stringent requirements set by tasks such as noninvasive in vivo sensing.

We thank M. Markham of Element Six for supplying the isotopically purified diamond sample, F. Aumayr and his team for implanting the sample with nitrogen ions, and K. Buczak for characterizing that sample. T. N. acknowledges the Graduate School for Complex Quantum Systems, funded by the Austrian Science Fund FWF, which also provided financial support to J.S. (the Wittgenstein Award). S. R. acknowledges financial support by FWF through Project No. F49-P10 (SFB NextLite). F. M. and B. B. acknowledge financial support by the ERC (ODYCQUENT). A. A. acknowledges support by FWF (Doctoral School "Building Solids for Function" Project No. W1243).

*Present address: Research Institute of Molecular Pathology and Department of Structural and Computational Biology, Research Platform Quantum Phenomena \& Nanoscale Biological Systems (QuNaBioS), University of Vienna, Dr. Bohr-Gasse 7, 1030 Vienna, Austria. tobias.noebauer@ati.ac.at

[1] Y. Kubo, C. Grezes, A. Dewes, T. Umeda, J. Isoya, H. Sumiya, N. Morishita, H. Abe, S. Onoda, T. Ohshima, V. Jacques, A. Dreau, J.-F. Roch, I. Diniz, A. Auffeves, D. Vion, D. Esteve, and P. Bertet, Phys. Rev. Lett. 107, 220501 (2011). 
[2] R. Amsüss, C. Koller, T. Nöbauer, S. Putz, S. Rotter, K. Sandner, S. Schneider, M. Schramböck, G. Steinhauser, H. Ritsch, J. Schmiedmayer, and J. Majer, Phys. Rev. Lett. 107, 060502 (2011).

[3] H. J. Kimble, Nature (London) 453, 1023 (2008).

[4] C. Brif, R. Chakrabarti, and H. Rabitz, New J. Phys. 12, 075008 (2010).

[5] V. F. Krotov, V. F. Krotov, and K. Krotov, Global Methods in Optimal Control Theory, Pure and Applied Mathematics Vol. 195 (Marcel Dekker, New York, 1995).

[6] T. E. Skinner, T. O. Reiss, B. Luy, N. Khaneja, and S. J. Glaser, J. Magn. Reson. 163, 8 (2003).

[7] P. Doria, T. Calarco, and S. Montangero, Phys. Rev. Lett. 106, 190501 (2011).

[8] R. Bücker, T. Berrada, S. van Frank, J.-F. Schaff, T. Schumm, J. Schmiedmayer, G. Jäger, J. Grond, and U. Hohenester, J. Phys. B 46, 104012 (2013).

[9] J. Scheuer, X. Kong, R. S. Said, J. Chen, A. Kurz, L. Marseglia, J. Du, P. R. Hemmer, S. Montangero, T. Calarco, B. Naydenov, and F. Jelezko, New J. Phys. 16, 093022 (2014).

[10] F. Dolde, V. Bergholm, Y. Wang, I. Jakobi, B. Naydenov, S. Pezzagna, J. Meijer, F. Jelezko, P. Neumann, T. SchulteHerbrüggen, J. Biamonte, and J. Wrachtrup, Nat. Commun. 5, 3371 (2014).

[11] B. Bartels and F. Mintert, Phys. Rev. A 88, 052315 (2013).

[12] G. Balasubramanian, I. Y. Chan, R. Kolesov, M. Al-Hmoud, J. Tisler, C. Shin, C. Kim, A. Wojcik, P. R. Hemmer, A. Krueger, T. Hanke, A. Leitenstorfer, R. Bratschitsch, F. Jelezko, and J. Wrachtrup, Nature (London) 455, 648 (2008).

[13] J. R. Maze, P. L. Stanwix, J. S. Hodges, S. Hong, J. M. Taylor, P. Cappellaro, L. Jiang, M. V. G. Dutt, E. Togan, A. S. Zibrov, A. Yacoby, R. L. Walsworth, and M. D. Lukin, Nature (London) 455, 644 (2008).

[14] F. Dolde, H. Fedder, M. W. Doherty, T. Nöbauer, F. Rempp, G. Balasubramanian, T. Wolf, F. Reinhard, L. C. L. Hollenberg, F. Jelezko, and J. Wrachtrup, Nat. Phys. 7, 459 (2011).

[15] G. Kucsko, P. C. Maurer, N. Y. Yao, M. Kubo, H. J. Noh, P. K. Lo, H. Park, and M. D. Lukin, Nature (London) 500, 54 (2013).

[16] D. Daems, A. Ruschhaupt, D. Sugny, and S. Guérin, Phys. Rev. Lett. 111, 050404 (2013).

[17] T. E. Skinner and N. I. Gershenzon, J. Magn. Reson. 204, 248 (2010).

[18] D. Abramovich and S. Vega, J. Magn. Reson., Ser. A 105, 30 (1993).

[19] V. M. Acosta, E. Bauch, M. P. Ledbetter, C. Santori, K.-M. C. Fu, P. E. Barclay, R. G. Beausoleil, H. Linget, J. F. Roch, F. Treussart, S. Chemerisov, W. Gawlik, and D. Budker, Phys. Rev. B 80, 115202 (2009).
[20] T. Nöbauer, K. Buczak, A. Angerer, S. Putz, G. Steinhauser, J. Akbarzadeh, H. Peterlik, J. Majer, J. Schmiedmayer, and M. Trupke, arXiv:1309.0453.

[21] E. Kim, V. M. Acosta, E. Bauch, D. Budker, and P. R. Hemmer, Appl. Phys. Lett. 101, 082410 (2012).

[22] S. Putz, D. O. Krimer, R. Amsüss, A. Valookaran, T. Nöbauer, J. Schmiedmayer, S. Rotter, and J. Majer, Nat. Phys. 10, 720 (2014).

[23] L. M. Pham, D. Le Sage, P. L. Stanwix, T. K. Yeung, D. Glenn, A. Trifonov, P. Cappellaro, P. R. Hemmer, M. D. Lukin, H. Park, A. Yacoby, and R. L. Walsworth, New J. Phys. 13, 045021 (2011).

[24] D. Le Sage, K. Arai, D. R. Glenn, S. J. DeVience, L. M. Pham, L. Rahn-Lee, M. D. Lukin, A. Yacoby, A. Komeili, and R. L. Walsworth, Nature (London) 496, 486 (2013).

[25] See Supplemental Material at http://link.aps.org/ supplemental/10.1103/PhysRevLett.115.190801, which includes Refs. [26-30], as well as additional methods, sample details, data tables, and smooth optimal control theory.

[26] G. Goelman, S. Vega, and D. B. Zax, Phys. Rev. A 39, 5725 (1989).

[27] M. Howard, J. Twamley, C. Wittmann, T. Gaebel, F. Jelezko, and J. Wrachtrup, New J. Phys. 8, 33 (2006).

[28] M. A. Nielsen and I. L. Chuang, Quantum Computation and Quantum Information, 10th ed. (Cambridge University Press, Cambridge, England, 2010).

[29] A. Gilchrist, N. K. Langford, and M. A. Nielsen, Phys. Rev. A 71, 062310 (2005).

[30] P. L. Stanwix, L. M. Pham, J. R. Maze, D. Le Sage, T. K. Yeung, P. Cappellaro, P. R. Hemmer, A. Yacoby, M. D. Lukin, and R. L. Walsworth, Phys. Rev. B 82, 201201 (2010).

[31] V. Jacques, P. Neumann, J. Beck, M. Markham, D. Twitchen, J. Meijer, F. Kaiser, G. Balasubramanian, F. Jelezko, and J. Wrachtrup, Phys. Rev. Lett. 102, 057403 (2009).

[32] M. H. Levitt, Composite pulses, eMagRes, doi:10.1002/ 9780470034590.emrstm0086 (2007).

[33] U. Hohenester, P. K. Rekdal, A. Borzi, and J. Schmiedmayer, Phys. Rev. A 75, 023602 (2007).

[34] D. Le Sage, L. M. Pham, N. Bar-Gill, C. Belthangady, M. D. Lukin, A. Yacoby, and R. L. Walsworth, Phys. Rev. B 85, 121202 (2012).

[35] J. M. Taylor, P. Cappellaro, L. Childress, L. Jiang, D. Budker, P. R. Hemmer, A. Yacoby, R. Walsworth, and M. D. Lukin, Nat. Phys. 4, 810 (2008).

[36] C. D. Aiello, M. Hirose, and P. Cappellaro, Nat. Commun. 4, 1419 (2013). 